\title{
Role of axillary ultrasound in the evaluation of early breast cancer in the era of Z0011: Time to redefine?
}

\author{
Corresponding author: \\ Abdul Syed \\ Southend University Hospital, \\ Prittlewell Chase, SSO ORY Southend \\ on Sea, United Kingdom, \\ e-mail: abdul.syed@southend.nhs.uk
}

Medical Research Journal 2018;

Volume 3, Number 2, 82-88

10.5603/MRJ.2018.0014

Copyright (C) 2018 Via Medica

ISSN 2451-2591

\begin{abstract}
Introduction: Ultrasound with concurrent histology of abnormal axillary lymph nodes has revolutionized the treatment of patients with breast cancer. By identifying nodal metastases, patients can avoid a two-stage axillary procedure. However, the results of the American College of Surgeons Oncology Group Z0011 trial indicate that a certain group of patients may have been over-treated with axillary dissection. Our aim was to analyze the nodal burden of patients identified by axillary ultrasound and to determine the proportion of patients who could have foregone axillary dissection incorporating Z0011 trial.

Methods: A retrospective analysis of patients with diagnosed breast cancer who underwent direct axillary dissection was performed. Based on nodal metastases patients were categorized into 'extensive' and 'minimal' groups and studied. Demographics and tumor characteristics were analyzed and eligibility for the Z0011 study was determined.

Results: All 1745 patients diagnosed with breast cancer underwent axillary ultrasound from April 2009 to March 2015. Of these, 197 patients had histology-proven nodal metastases and underwent direct axillary lymph node dissection. One hundred and twenty-one patients (61.4\%) had extensive and 76 patients (38.6\%) had minimal nodal metastases. Of the latter, 23 patients (11.7\%) fulfilled the Z0011 criteria. Conclusion: This study demonstrated that a large proportion of patients had minimal nodal involvement (38.6\%), in contrast to the results published in the literature. In addition, a significant number of patients could have avoided axillary dissection (11.7\%) based on the Z0011 criteria. Hence our study encourages to redefine the role of axillary ultrasound to avoid unnecessary axillary dissection.

Key points:

1. Axillary ultrasound is performed in all patients diagnosed with breast cancer.

2. Axillary ultrasound findings do not correlate with histological burden of nodal disease.

3. Role of axillary ultrasound needs to be redefined to avoid unnecessary axillary dissection. Key words: Ultrasonography; Cytology; Metastasis; Breast Cancer; Lymph nodes
\end{abstract}

Med Res J 2018; 3 (2): 82-88

\section{Introduction}

Axillary ultrasound combined with fine needle aspiration cytology (FNAC) or core biopsy (CB) has established its role as an accurate tool for identifying axillary nodal metastasis. This procedure identifies approximately $50 \%$ of nodal involvement pre-operatively [1]. In the United Kingdom, axillary ultrasound is customarily performed for all patients diagnosed with breast cancer, as recommended by the National Institute for Health and Care Excellence (NICE) [2]. If nodal involvement is detected by a combination of ultrasound and $\mathrm{FNAC/CB}$, direct axillary lymph node dissection (ALND) is performed.
If axillary ultrasound is unable to identify nodal metastatic disease, sentinel lymph node biopsy will be performed as the gold standard for staging of axillary disease [3-4]. ALND is then recommended as a separate procedure if the sentinel node biopsy yields a positive result [5-6]. Axillary ultrasound therefore facilitates patients to undergo a direct single stage axillary dissection rather than this two-stage procedure. This reduces patient morbidity and generates financial benefits for the healthcare system [7]. However, axillary ultrasound may prove to be a double-edged sword, leading to an over-treatment of axillary disease associated with its significant morbidities [8-9]. This is because various studies have demonstrated that the sentinel lymph node 
was the only positive lymph node in $40-60 \%$ of patients [10-11] and also nomograms have been validated to identify patients who are at risk of non-sentinel lymph node metastasis [12-14]. More importantly, the results of the American College of Surgeons Oncology Group (ACOSOG) Z0011 trial published earlier in 2011 and recently updated in 2017 suggest that a subgroup of patients can safely avoid ALND after positive sentinel lymph node biopsy [15-16]. In this trial, patients with a tumor size less than $5 \mathrm{~cm}$ who also had one or two positive sentinel nodes (without matting or extra nodal extension) were treated with breast-conserving surgery and whole breast irradiation. This study had a median follow up of 9.25 years and found no difference in the overall survival [15] or locoregional recurrence [16] in patients who did or did not undergo axillary lymph node dissection after a positive sentinel lymph node biopsy. Despite some limitations [17-18], this study is the best available evidence to date with long term follow up, supporting the avoidance of ALND in a sub-group of patients [19-20].

The primary aim of our study was to assess the axillary nodal burden from the histopathological findings of direct ALND and classify them into patients with minimal nodal involvement ( $\leq 2$ positive nodes) and extensive nodal involvement ( $>2$ positive nodes). This study also aimed to identify which of our patients could have safely foregone ALND based on the findings of the Z0011 trial. Overall, we hoped to find features which may be predictive of minimal or extensive nodal involvement by analyzing the differences in tumor characteristics and outcomes between the two groups.

\section{Material and methods}

Patients with primary breast cancer between April 2009 and March 2015 were identified from the surgical database of our hospital and retrospectively analyzed. Our departmental policy was to perform axillary ultrasound on all patients with suspected breast cancer. Radiologists with an interest in breast, as well as trained clinical specialists, performed the axillary ultrasounds. Lymph nodes were deemed suspicious if they were multiple and large $(>1 \mathrm{~cm})$, with eccentric or more than $2 \mathrm{~mm}$ cortical thickening and replacement or loss of the fatty hilum.

Suspicious nodes identified using axillary ultrasound were sampled using FNAC or CB, at the operator's discretion. Histopathological assessment of nodal samples was then performed using hematoxylin and eosin staining and an immunohistochemical profile was then generated upon identification of metastatic disease. Patients with axillary nodal metastatic disease underwent direct ALND, accompanied by wide local excision (WLE) or mastectomy as deemed clinically appropriate.

Details regarding positive nodal disease from FNAC/CB following axillary ultrasound and further ALND were obtained from histopathological reports. Demographics and tumor characteristics were identified from multi-disciplinary discussions and clinical letters were used to follow-up significant events.

Histopathological data collected were size, type and grade of tumor, multifocality, lymphovascular invasion, HER2 status and the presence of estrogen and progesterone receptors. The number of nodes removed during ALND were recorded as well as the number of positive nodes to categorize into 'minimal' and 'extensive' groups.

We then identified which of these patients could have safely foregone axillary lymph node dissection based on the Z0011 criteria. This included patients who underwent breast conserving surgery for a tumor size less than $5 \mathrm{~cm}$, had $\leq 2$ positive nodes from axillary dissection without matting and extra-nodal extension and were treated with adjuvant whole breast irradiation. Patients who underwent preoperative neo-adjuvant treatment and who had a mastectomy as the definitive treatment were excluded.

\section{Statistical analyses}

All analyses were conducted with The Graph Pad software (Prism version 5). For numeric data, values are expressed in median with percentages and Chi squared analyses or a Fisher's exact test were used to assess differences in patient and tumor characteristics between those with minimal nodal involvement $(\leq 2$ positive nodes) and extensive nodal involvement ( $>2$ positive nodes). A $p$ value of $\leq 0.05$ was considered significant.

\section{Results}

A total of 1745 patients were diagnosed with operable breast cancer between April 2009 and March 2015. Of these, 269 patients underwent direct ALND for axillary nodal metastases. Ten patients had undergone axillary dissection for recurrent breast cancer and were excluded. Twenty patients were excluded from the study because there were no clear indications for ALND in place of sentinel lymph node biopsy. Biopsies were not performed in 14 patients due to technical reasons, although these patients were highly suspicious for nodal metastases from axillary ultrasound. Three patients had an axillary dissection based on an abnormality in another modality of imaging (CT, MRI and mammogram). Another four patients had axillary dissection based on 
suspicion of nodal metastases intraoperatively. Twenty-one patients had benign findings on FNAC/CB, but still received axillary dissection due to high suspicion from axillary ultrasound and were thus excluded.

In total, seventy-two patients were excluded from the study, and the remaining 197 patients with definitive diagnosis of axillary nodal metastases on ultrasound and FNAC/CB who underwent direct ALND were ultimately considered eligible for the study as shown in Table 1.

The median age of the patients was 62 years (range, 23-92). All except one patient were female. Eightyfive patients $(43.1 \%)$ had breast-conserving surgery, while 112 patients (56.9\%) underwent mastectomy. The median pathological tumor size was $24.5 \mathrm{~mm}$ (range, $5-130 \mathrm{~mm}$ ). Fifty six of the 197 patients $(28.4 \%)$ had T1 tumors, and 119 patients (60.4\%) had T2 tumors. Seventeen patients $(8.6 \%)$ had a tumor greater than $50 \mathrm{~mm}$, while multifocal disease was detected in 12 patients $(6 \%)$. Invasive ductal carcinoma was the predominant tumor upon final pathology (172 patients, $87 \%$ ), while invasive lobular carcinoma was observed in 23 patients (12\%). Other pathologies were noted in two patients (medullary carcinoma).

Grade 3 disease was found in 136 patients (69\%), 57 patients had grade 2 and only four patients had grade 1 disease. Lymphovascular invasion was present in 103 patients ( $n=193,53.4 \%$ ). The majority of patients were estrogen receptor (ER) positive (155, $78.7 \%$ ), while 55 (27.9\%) were HER2 positive. Thirty-six patients (18.3\%) were ER positive/HER2 positive, while 23 patients $(11.7 \%)$ were triple negative.

Of the 197 patients, FNAC was diagnostic in 92 patients (C5 - 85; C4 - 5; C3 - 2), and a core biopsy confirmed metastases in the remaining 105 patients (B5 - 104; B4 -1). The median number of lymph nodes excised during axillary dissection was 15 (range, 4-35), and the median number of positive nodes was 3 (range, 0-31). Five patients had no positive nodes upon axillary dissection. Within this group of patients, 76 patients (38.6\%) had two positive nodes or less from ALND (minimal nodal metastases), and 121 patients had more than two positive nodes (extensive nodal metastases). The characteristics of these groups of patients are outlined in Table 1.

There was no significant difference observed between the two groups in terms of age, type of surgery, tumor size, morphology, estrogen receptor status, HER2 status and multifocality. Differences in tumor grade, progesterone and triple-negative receptor status were borderline but not significant. However, the presence of lymphovascular invasion was significant in patients with extensive nodal metastasis $(P<0.001)$.

The median follow-up was 4.6 years. A total of 15 patients died during follow up; eight from the minimal
Table 1. Characteristics of patients who had axillary clearance and grouped into minimal nodal and extensive nodal metastases

\begin{tabular}{lccc}
\hline $\begin{array}{l}\text { Patient } \\
\text { characteristics }\end{array}$ & $\begin{array}{c}\text { Positive } \\
\text { lymph } \\
\text { nodes } \\
(n=76)\end{array}$ & $\begin{array}{c}\text { Positive } \\
\text { lymph } \\
\text { nodes } \\
(n=121)\end{array}$ \\
\hline
\end{tabular}

Age
Median (Range)
$<50$ years
$50-69$ years
$=/>70$ years
Type of surgery
Breast conserving

Mastectomy

$\begin{array}{cc}62(23-90) & 62(33-92) \\ 15(19.7 \%) & 32(26.4 \%) \\ 37(48.7 \%) & 49(40.5 \%) \\ 24(31.6 \%) & 40(33.1 \%)\end{array}$

0.44

Tumor size in $\mathbf{m m}$

Median (Range)

$<20 \mathrm{~mm}$

20-30 mm

$>30 \mathrm{~mm}$

$38(50.0 \%) \quad 47(38.8 \%)$

$38(50.0 \%) \quad 74(61.2 \%)$

0.14

\section{Morphology}

of tumor

Ductal carcinoma

Lobular carcinoma

Other types

$23(5-60) \quad 26(8-130)$

0.31

$23(30.3 \%) \quad 33(27.3 \%)$

$35(46.0 \%) \quad 44(36.4 \%)$

$18(23.7 \%) \quad 39(32.2 \%)$

$\begin{array}{cc}70(92.1 \%) & 102(84.3 \%) \\ 5(6.6 \%) & 18(14.9 \%) \\ 1(1.3 \%) & 1(0.8 \%)\end{array}$

\section{Tumor Grade}

Grade 1

Grade 2

Grade 3

$3(3.9 \%) \quad 1(0.8 \%)$

$23(30.3 \%) \quad 34(28.1 \%)$

$50(65.8 \%) \quad 86(71.1 \%)$

$\mathrm{ER}^{*}$ status

Negative

Positive

$17(22.4 \%) \quad 25(20.7 \%)$

$59(77.6 \%) \quad 96(79.3 \%)$

0.86

$\mathrm{PR}^{\text {**}}$ status

Negative

Positive

$37(48.7 \%) \quad 49(40.5 \%)$

$39(51.3 \%) \quad 72(59.5 \%)$

0.30

Her2*** Status

Negative

Positive

$57(75 \%)$

$85(70.2 \%)$

Triple Negative

No

65 (85.5\%) 109 (90.1\%)

0.37

Yes

$11(14.5 \%) \quad 12(9.9 \%)$

Multifocality

No

$70(92.1 \%) \quad 115(95 \%)$

0.54

Yes

$6(7.9 \%) \quad 6(5 \%)$

Lymphovascular invasion

No

Yes

Not recorded

$52(68.4 \%)$

$24(31.6 \%)$

$51(42.1 \%)$

$66(54.5 \%)$

4

Mortality follow up

4-5yrs

Dead

$7(5.8 \%)$

0.27
$68(89.5 \%) \quad 114(94.2 \%)$

${ }^{*}$ ER - Estrogen Receptor

**PR - Progesterone Receptor

***Her2 - Human Epidermal Growth Factor Receptor 


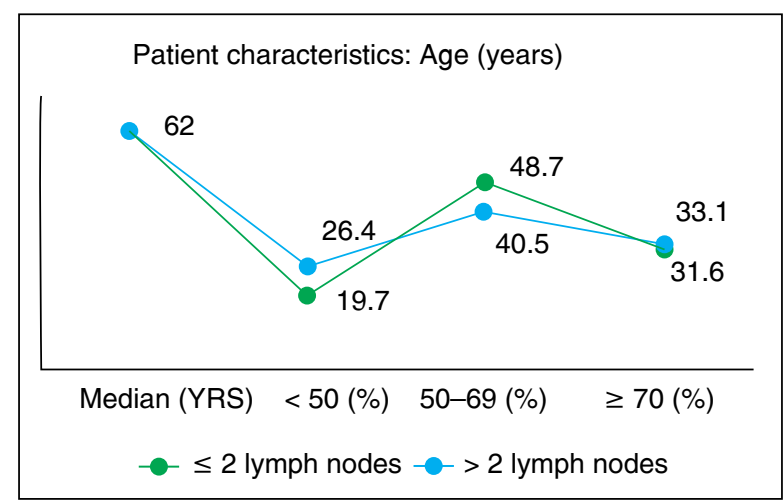

Figure 1. Patient characteristics: distribution with respect to age

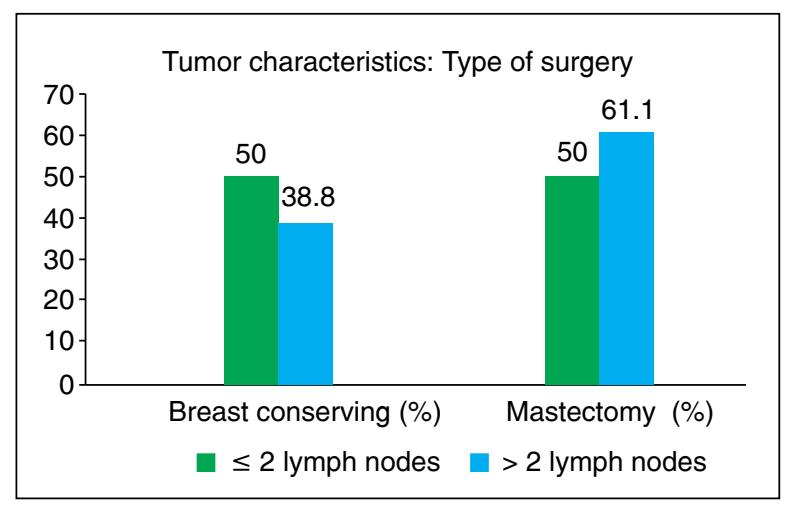

Figure 2. Tumor characteristics: distribution with respect to type of surgery

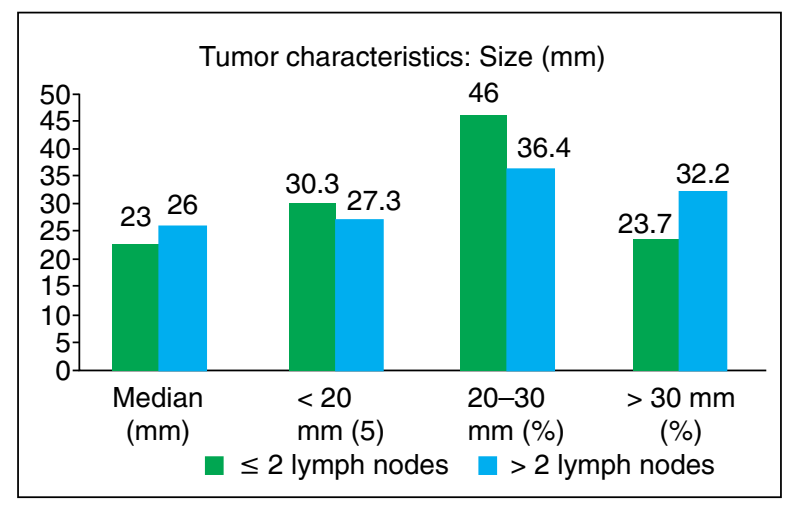

Figure 3. Tumor characteristics: distribution with respect to size of tumor

nodal group and seven from the extensive nodal group with an overall survival rate of $89.5 \%$ and $94.2 \%$, respectively. There was no statistical significance between the two groups in terms of survival.

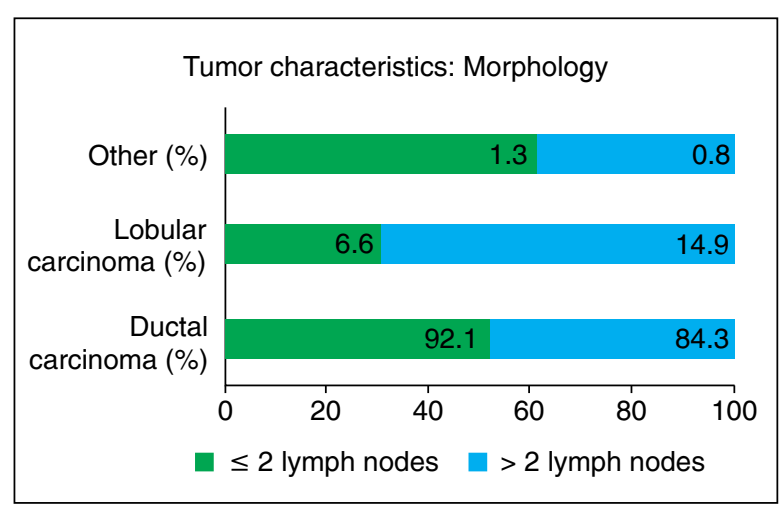

Figure 4. Tumor characteristics: distribution with respect to tumor morphology

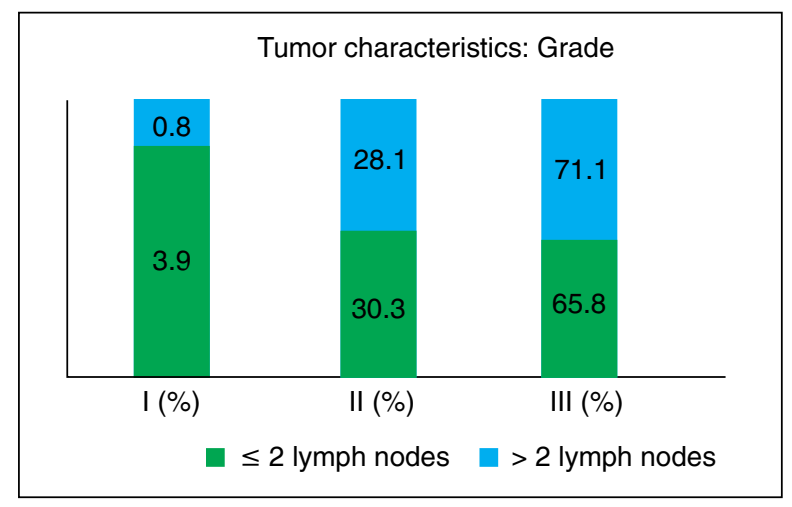

Figure 5. Tumor characteristics: distribution with respect to grade of tumor

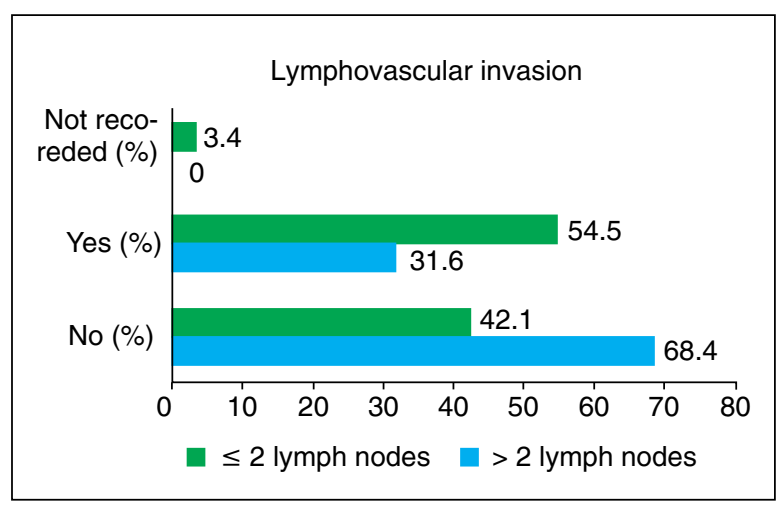

Figure 6. Tumor characteristics: distribution with respect to presence of lymphovascular invasion

Patients with minimal nodal involvement were potentially eligible for the Z0011 study. Of these, 53 patients were excluded as they did not meet the criteria, the predominant cause being mastectomy as the definitive 
breast surgery. The remaining 23 patients $(11.7 \%)$ satisfied the criteria for the Z0011 study, and could therefore have been spared axillary lymph node dissection.

\section{Discussion}

With advancement in technology and expertise in image interpretation, ultrasound has become a significant preoperative tool in the evaluation of nodal disease.

Studies have shown that high-resolution ultrasound can detect nodal metastases in breast cancer with a sensitivity and specificity ranging from $50-70 \%$ and 90-97.2\%, respectively [21]. In conjunction with cytology/histology, the literature demonstrates increase in sensitivity and specificity to $80-90 \%$ and $100 \%$, respectively, when conducted by experienced radiologists [22-23].

The improvement in ultrasound examination suggests that the chance of finding axillary metastases will increase. This means that more patients who would have otherwise had a sentinel lymph node biopsy will instead be subjected to ALND with its associated morbidity.

In our study, there were no significant differences observed when comparing the tumor characteristics between the two groups. The exception was lymphovascular invasion, which was predictive of patients with extensive nodal metastases. This histopathological factor needs to be taken into consideration while deciding on ALND following positive sentinel node biopsy regardless of the number of positive nodes.

There was no significant difference in overall survival between patients with minimal and extensive nodal metastasis. It is difficult to be certain whether the comparable survival rate achieved translates from both groups receiving ALND or being a simple chance finding needs to be determined.

Previous studies have predominantly shown a high axillary nodal burden in patients identified from axillary ultrasound [24-26]. However, in contrast, our study showed that more than one third of patients had minimal node involvement (38.6\%). These findings are in concurrent with the results obtained by Boland et al. [30], who also reports $38.6 \%$ of patients had less than three positive nodes from ALND. Selection bias may account for the high axillary nodal burden documented in the literature. Verheuvel et al. [24] explained that axillary ultrasound was performed by general radiologists who were not specialized in breast cancer and Caudle et al. [25] reported that, from 2010 , sentinel node-positive patients did not undergo axillary dissection and were excluded from their study. Reyna et al. [26] reported that axillary ultrasound was selectively performed for T2 or larger tumors since 2007 and that T1 tumors were evaluated according to the preference of the surgeon or clinical suspicion. These findings might explain the high axillary nodal burden documented in the literature. Therefore, based on our study, patients with clinically early-stage breast cancer do not have a significant axillary burden of disease when they were identified by axillary ultrasound with cytology / histology. Similar findings were observed by Cools et al. [27], who reports that patients with ultrasound guided biopsy do not harbor more nodal metastases than who are not subjected to it. Recent meta-analysis by Muneer et al. [28] concurred that $43 \%$ of patients with positive sentinel lymph node biopsy (SLNB) were subjected to unnecessary ALND.

Based on the findings of the Z0011 trial, a significant number of patients would have avoided direct axillary dissection (11.7\% over a period of six years) if they had undergone a sentinel lymph node biopsy rather than an ultrasound guided FNAC/CB. This is twice the number quoted in study by Boland et al. [29] likely due to more than half the number of patients undergoing mastectomy in their group.

Additional detail provided on ultrasound, such as the number of nodes involved and radiographic features of these nodes may further help to identify the patients who would benefit from a direct axillary lymph node dissection and those patients who should receive sentinel lymph node biopsy. Pilewskie et al. [30] stated that $68 \%$ of patients with more than one abnormal lymph node on ultrasound axilla have more than or equal to three nodal metastases on ALND. Further research, looking at the correlation between radiographic data and axillary disease burden, may further help to clarify the patients who would be candidates for sentinel lymph node biopsy. Radiologists should also be encouraged to clearly report the abnormalities detected on ultrasound axilla in the light of future studies.

The limitations of our study include biases acquired from retrospective analyses and difficulty in reading ultrasound reports. This is because of absence of precise documentations on ultrasound findings of the axilla in relation to number of lymph nodes as there is tendency to perform biopsy once abnormal node is identified. Although the sample size of our study is relatively small, it strongly advocates that more than one third of patients had low-volume nodal metastases, questioning the need for ALND in all patients with ultrasound guided biopsy proven nodal metastases.

Further prospective studies with novel concepts are required to fully ascertain the sub-group of patients who can forego axillary lymph node dissection. This could be by proceeding directly to SLNB without ultrasound guided biopsy for patients with single abnormal node or by clipping the node after positive biopsy and performing SLNB along with excision of clipped node rather than ALND. Therefore, in the era of Z0011, the role of axillary ultrasound needs to be redefined to avoid unnecessary axillary dissection. 


\section{Conclusions}

Ultrasound with concurrent histology of abnormal axillary lymph nodes is currently treated with direct ALND and can avoid two stage surgical procedure. However, our study shows that more than one third of these patients were over-treated and could have spared ALND. Hence the role of ultrasound axilla in early breast cancer needs to be redefined.

\section{List of Abbreviations:}

$\begin{array}{ll}\text { FNAC } & \text { Fine Needle Aspiration Cytology } \\ \text { CB } & \text { Core Biopsy } \\ \text { NICE } & \begin{array}{l}\text { National Institute for Health and Care Ex- } \\ \text { cellence }\end{array} \\ \text { ALND } & \text { Axillary Lymph Node Dissection } \\ \text { ACOSOG } & \begin{array}{l}\text { American College of Surgeons Oncology } \\ \text { Group }\end{array} \\ \text { WLE } & \text { Wide Local Excision } \\ \text { HER2 } & \text { Human Epidermal Growth Factor Receptor 2 } \\ \text { ER } & \text { Estrogen receptor } \\ \text { PR } & \text { Progesterone Receptor } \\ \text { SLNB } & \text { Sentinel Lymph Node Biopsy }\end{array}$

\section{References}

1. Diepstraten SCE, Sever AR, Buckens CFM, et al. Value of preoperative ultrasound-guided axillary lymph node biopsy for preventing completion axillary lymph node dissection in breast cancer: a systematic review and meta-analysis. Ann Surg Oncol. 2014; 21(1): 51-59, doi: 10.1245/s10434-013-3229-6, indexed in Pubmed: 24008555.

2. National Collaborating Centre for Cancer UK. Early and locally advanced breast cancer: diagnosis and treatment. NICE guidelines [CG80] Published date. 2009: February.

3. Mansel RE, Fallowfield L, Kissin M, et al. Randomized multicenter trial of sentinel node biopsy versus standard axillary treatment in operable breast cancer: the ALMANAC Trial. J Natl Cancer Inst. 2006; 98(9): 599-609, doi: 10.1093/jnci/djj158, indexed in Pubmed: 16670385

4. Veronesi U, Viale G, Paganelli G, et al. Sentinel lymph node biopsy in breast cancer: ten-year results of a randomized controlled study. Ann Surg. 2010; 251(4): 595-600, doi: 10.1097/SLA.0b013e3181c0e92a, indexed in Pubmed: 20195151.

5. Krag DN, Anderson SJ, Julian TB, et al. Sentinel-lymph-node resection compared with conventional axillary-lymph-node dissection in clinically node-negative patients with breast cancer: overall survival findings from the NSABP B-32 randomised phase 3 trial. Lancet Oncol. 2010; 11(10): 927-933, doi: 10.1016/S1470-2045(10)70207-2, indexed in Pubmed: 20863759.

6. Lyman GH, Giuliano AE, Somerfield MR, et al. American Society of Clinical Oncology. American Society of Clinical Oncology guideline recommendations for sentinel lymph node biopsy in early-stage breast cancer. J Clin Oncol. 2005; 23(30): 7703-7720, doi: 10.1200/JCO.2005.08.001, indexed in Pubmed: 16157938.

7. Boughey JC, Moriarty JP, Degnim AC, et al. Cost modeling of preoperative axillary ultrasound and fine-needle aspiration to guide surgery for invasive breast cancer. Ann Surg Oncol. 2010; 17(4): 953-958, doi: 10.1245/s10434-010-0919-1, indexed in Pubmed: 20127185

8. Ashikaga T, Krag DN, Land SR, et al. National Surgical Adjuvant Breast, Bowel Project. Morbidity results from the NSABP B-32 trial comparing sentinel lymph node dissection versus axillary dissection. J Surg Oncol. 2010; 102(2): 111-118, doi: 10.1002/jso.21535, indexed in Pubmed: 20648579.

9. Goyal A, Newcombe RG, Chhabra A, et al. Morbidity in breast cancer patients with sentinel node metastases undergoing delayed axillary lymph node dissection (ALND) compared with immediate ALND. Ann Surg Oncol. 2008; 15(1): 262-267, doi: 10.1245/s10434-007-9593-3, indexed in Pubmed: 17879117.

10. Grube BJ, Giuliano AE. Observation of the breast cancer patient with a tumor-positive sentinel node: implications of the ACOSOG Z0011 trial. Semin Surg Oncol. 2001; 20(3): 230-237, indexed in Pubmed: 11523108.

11. Coutant $\mathrm{C}$, Morel $\mathrm{O}$, Antoine $\mathrm{M}$, et al. [Is axillary lymph node dissection always necessary in breast cancer patients with a positive sentinel node?]. J Chir (Paris). 2007; 144(6): 492-501, indexed in Pubmed: 18235360.

12. Gur AS, Unal B, Ozbek U, et al. Turkish Federation of Breast Disease Associations Protocol MF08-01 investigators. Validation of breast cancer nomograms for predicting the non-sentinel lymph node metastases after a positive sentinel lymph node biopsy in a multi-center study. Eur J Surg Oncol. 2010; 36(1): 30-35, doi: 10.1016/j.ejso.2009.05.007, indexed in Pubmed: 19535217.

13. Unal B, Gur AS, Kayiran O, et al. Models for predicting non-sentinel lymph node positivity in sentinel node positive breast cancer: the importance of scoring system. Int J Clin Pract. 2008; 62(11): 1785-1791, doi: 10.1111/j.1742-1241.2008.01887.x, indexed in Pubmed: 19143863.

14. Hessman CJ, Naik AM, Kearney NM, et al. Comparative validation of online nomograms for predicting nonsentinel lymph node status in sentinel lymph node-positive breast cancer. Arch Surg. 2011; 146(9): 1035-1040, doi: 10.1001/archsurg.2011.201, indexed in Pubmed: 21931000.

15. Giuliano AE, Ballman KV, McCall L, et al. Effect of Axillary Dissection vs No Axillary Dissection on 10-Year Overall Survival Among Women With Invasive Breast Cancer and Sentinel Node Metastasis: The ACOSOG Z0011 (Alliance) Randomized Clinical Trial. JAMA. 2017; 318(10): 918926, doi: 10.1001/jama.2017.11470, indexed in Pubmed: 28898379.

16. Giuliano AE, Ballman K, McCall L, et al. Locoregional Recurrence After Sentinel Lymph Node Dissection With or Without Axillary Dissection in Patients With Sentinel Lymph Node Metastases: Long-term Follow-up From the American College of Surgeons Oncology Group (Alliance) ACOSOG Z0011 Randomized Trial. Ann Surg. 2016; 264(3): 413-420, doi $10.1097 /$ SLA 0000000000001863 , indexed in Pubmed: 27513155.

17. Shah-Khan M, Boughey JC. Evolution of axillary nodal staging in breast cancer: clinical implications of the ACOSOG Z0011 trial. Cancer Control. 2012; 19(4): 267-276, doi: 10.1177/107327481201900403, indexed in Pubmed: 23037494.

18. Reznik J, Cicchetti MG, Degaspe B, et al. Analysis of axillary coverage during tangential radiation therapy to the breast. Int $\mathrm{J}$ Radiat Oncol Biol Phys. 2005; 61(1): 163-168, doi: 10.1016/j.jirobp.2004.04.065, indexed in Pubmed: 15629607.

19. Caudle AS, Hunt KK, Mittendorf EA, et al. Multidisciplinary considerations in the implementation of the findings from the American College of Surgeons Oncology Group (ACOSOG) Z0011 study: a practice-changing trial. Ann Surg Oncol. 2011; 18(9): 2407-2412, doi: 10.1245/s10434-011-1593-7, indexed in Pubmed: 21327455.

20. Yi M, Kuerer HM, Mittendorf EA, et al. Impact of the american college of surgeons oncology group Z0011 criteria applied to a contemporary patient population. J Am Coll Surg. 2013; 216(1): 105-113, doi: 10.1016/j.jamcollsurg.2012.09.005, indexed in Pubmed: 23122536.

21. Motomura K, Inaji H, Komoike Y, et al. Gamma probe and ultrasonographically-guided fine-needle aspiration biopsy of sentinel lymph nodes in breast cancer patients. Eur J Surg Oncol. 2001; 27(2): 141-145, doi: 10.1053/ejso.2000.1059, indexed in Pubmed: 11289748.

22. van Wely BJ, de Wilt JHW, Schout PJC, et al. Ultrasound-guided fine-needle aspiration of suspicious nodes in breast cancer patients; selecting patients with extensive nodal involvement. Breast Cancer Res Treat. 2013; 140(1): 113-118, doi: 10.1007/s10549-013-2624-9, indexed in Pubmed: 23813331.

23. Houssami N, Diepstraten SCE, Cody HS, et al. Clinical utility of ultrasound-needle biopsy for preoperative staging of the axilla in invasive breast cancer. Anticancer Res. 2014; 34(3): 1087-1097, indexed in Pubmed: 24596347

24. Verheuvel NC, van den Hoven I, Ooms HWA, et al. The role of ultrasound-guided lymph node biopsy in axillary staging of invasive breast cancer in the post-ACOSOG Z0011 trial era. Ann Surg Oncol. 2015; 22(2): 409-415, doi: 10.1245/s10434-014-4071-1, indexed in Pubmed: 25205303.

25. Caudle AS, Kuerer HM, Le-Petross HT, et al. Predicting the extent of nodal disease in early-stage breast cancer. Ann Surg Oncol. 2014; 21(11): 3440-3447, doi: 10.1245/s10434-014-3813-4, indexed in Pubmed: 24859939.

26. Reyna C, Lee MC, Frelick A, et al. Axillary burden of disease following false-negative preoperative axillary evaluation. Am J Surg. 2014; 208(4): 577-581, doi: 10.1016/j.amjsurg.2014.05.015, indexed in Pubmed: 25118162 
27. Cools-Lartigue J, Sinclair A, Trabulsi N, et al. Preoperative axillary ultrasound and fine-needle aspiration biopsy in the diagnosis of axillary metastases in patients with breast cancer: predictors of accuracy and future implications. Ann Surg Oncol. 2013; 20(3): 819-827, doi: 10.1245/s10434-012-2609-7, indexed in Pubmed 22972506

28. Ahmed M, Jozsa F, Baker R, et al. Meta-analysis of tumour burden in pre-operative axillary ultrasound positive and negative breast cancer patients. Breast Cancer Res Treat. 2017; 166(2): 329-336, doi 10.1007/s10549-017-4405-3, indexed in Pubmed: 28755147.
29. Boland MR, Prichard RS, Daskalova I, et al. Axillary nodal burden in primary breast cancer patients with positive pre-operative ultrasound guided fine needle aspiration cytology: management in the era of ACOSOG Z011. Eur J Surg Oncol. 2015; 41(4): 559-565, doi: 10.1016/j. ejso.2015.01.011, indexed in Pubmed: 25648466.

30. Pilewskie M, Mautner SK, Stempel M, et al. Does a Positive Axillary Lymph Node Needle Biopsy Result Predict the Need for an Axillary Lymph Node Dissection in Clinically Node-Negative Breast Cancer Patients in the ACOSOG Z0011 Era? Ann Surg Oncol. 2016; 23(4): 1123-1128, doi: 10.1245/s10434-015-4944-y, indexed in Pubmed: 26553439 